\title{
Common and specific features in pictorial analogies
}

\author{
IN-MAO LIU \\ National Taiwan University, Taipei, Taiwan, Republic of China
}

\begin{abstract}
Pictures belonging to different domains (cups and persons), as well as pictures belonging to the same domains (cups or persons only), were used to construct analogies of various paradigms. Pictures from different domains shared three common features, while pictures within each domain had two features specific to that domain. When no specific feature change was introduced, an asymmetry of the paradigms (A cup is to B cup as $R$ person is to $S$ person and $P$ person is to $Q$ person as $C$ cup is to $D$ cup) was obtained as an extension of the theory of metaphoricity (Ortony, 1979) predicted. When specific feature changes were introduced, however, the obtained results were not as the extended theory would predict. An alternative model that emphasizes the function of a set of reference feature changes in extraction and comparison of the feature changes that may exist in the first and second terms was proposed to account for the experimental findings.
\end{abstract}

Verbal or geometric analogies generally take the form "A is to $B$ as $C$ is to $D "(A: B:: C: D)$. In the laboratory or in psychometric tests, analogies are tested in two formats: true-false analogies and forced-choice analogies. In true-false analogies, the subject tries to determine whether a relation can be constructed from $\mathrm{C}$ to $\mathrm{D}$ that is analogous to the relation from $\mathrm{A}$ to $\mathrm{B}$. In forcedchoice analogies $\left(A: B:: C: D_{1}, D_{2}\right)$, the subject tries to determine which alternative $\left(D_{1}\right.$ or $\left.D_{2}\right)$ best forms a $\mathrm{C}$-to-D relation that is analogous to the A-to-B relation.

Most models of analogical reasoning (e.g., Evans, 1968; Grudin, 1980; Mulholland, Pellegrino, \& Glaser, 1980; Sternberg, 1977; Whitely \& Barnes, 1979) agree on the following processes of solving an analogy. First, the geometric patterns comprising the $\mathrm{A}$ and $\mathrm{B}$ terms of the analogy are decomposed into subpatterns or elements, and the transformations that relate the elements in the A-B pair of terms are determined. (In the case of verbal analogies, the $\mathrm{A}$ and $\mathrm{B}$ terms are decomposed into features.) Second, the same process applies to the C-D pair of terms. Third, the A-B transformations and the C-D transformations are compared to determine the correctness of the D term.

Several important issues raised are as follows. The first issue is concerned with how the relation between the $A$ and $C$ terms affects solution time. Sternberg(1977) postulated that extraction of the A.C relation is a necessary condition for solving an analogy. Hence, if there is a larger difference between $\mathrm{A}$ and $\mathrm{C}$ in terms of

This study was supported by National Science Council Grant NSC-69H-03-02(03), Republic of China. The author is grateful to Chu-hung Chao and Ching-ching Ting for their assistance in collecting the data and to Robert $\mathbf{J}$. Sternberg and Susan E. Whitely for their helpful comments on an earlier version of this manuscript. Requests for reprints should be sent to In-mao Liu, Department of Psychology, National Taiwan University, Taipei, Taiwan, Republic of China. number of features changed from $\mathrm{A}$ to $\mathrm{C}$, then the solution time is longer. Sternberg's model encounters a logical difficulty because the $\mathrm{A}$ and $\mathrm{C}$ terms may be almost totally unrelated (almost an infinite number of features changed from the $\mathrm{A}$ to the $\mathrm{C}$ term). However, an analogy of this sort may be solved as fast as one in which the $\mathrm{A}$ and $\mathrm{C}$ terms are closely related. An example is "diamond : gem : : elm : ?", in which "diamond" and "elm" are almost unrelated. Nevertheless, it is easy to find the solution. Essentially based on this observation, Grudin (1980) posits that the subject switches to examination of the relationship between the $\mathrm{A}$ and $\mathrm{C}$ terms only when there is no success in finding the relationship between the $A$ and $B$ terms. In view of the experimental findings of Grudin and Sternberg, it may be concluded that the subject attempts to identify the A.C transformations when it is very difficult or impossible to find the A-B transformations and then proceeds to compare the A-C and B-D transformations to determine the correctness of the D term. Furthermore, when it is possible to find the A-B transformations, the subject's solution attempt will be facilitated to the extent that the $\mathrm{A}$ and $\mathrm{C}$ terms are related, because the A-C relationship facilitates identification of the C-D transformations or/and comparison of the A-B and C-D transformations.

The second issue is concerned with how the information structure of individual analogy terms affects the solution time. With respect to verbal analogies, the information structure of individual analogy terms is difficult to identify. Mulholland et al. (1980) used simple geometric elements such as a line, triangle, circle, cross, rectangle, and pentagon to construct geometric analogies by combining one to three elements as individual terms. They found that increasing the number of elements systematically increased time to solution. This suggests that the patterns comprising the 
terms of the analogies were decomposed serially, element by element, as they hypothesized.

\section{Major Problem Raised}

The present study is primarily concerned with the second issue of how the information structure of individual analogy terms affects solution time. In the case of geometric analogies, it is easy to decompose individual patterns into subpatterns or elements exhaustively. Hence, solution to a geometric analogy may proceed almost mechanically. With respect to verbal analogies, however, extracting features from individual analogy terms and then finding the number of features changed from $A$ to $B$ or from $C$ to $D$ is not easy to accomplish. Let us consider the same example of Sternberg (1977),

\section{WASHINGTON : LINCOLN $:: 1: 10,5$.}

In attempting to solve this analogy according to the existing models, the subject may extract many features associated with Washington and Lincoln. Their walking gestures, their temperaments, and their attitudes toward subordinates are only a few of such features. Indeed, such features are nearly infinite. The subject then engages himself in identifying how a particular feature changes from Washington to Lincoln. If a deliberate subject encodes a variety of such features, any model that claims an exhaustive process in feature extraction or transformation identification for the $\mathrm{A}$ and $\mathrm{B}$ terms would have difficulty in accounting for usually short solution time for a verbal analogy. Even if a model is revised to allow a self-terminating process, searching for a correct feature out of nearly infinite possible features riy frustrate the subject before he hits upon the solution.

in attempting to solve a verbal analogy, then, the subject must be able to disregard irrelevant features and concentrate on only those features that are most pertinent, in the eye of the subject, to a solution. How is the subject able to discriminate those features that are relevant from those features that are irrelevant? Obviously, the subject tries to extract those features changed from the $A$ to the $B$ term that are also changeable from the $C$ to the $D$ term. Take the WashingtonLincoln analogy, for instance: One can identify a transformation from the $\mathrm{A}$ to the $\mathrm{B}$ term on the basis of a change in the feature of their walking gestures. However, it is impossible to find any analogous transformation based on this feature for the C and D terms. Since the $C$ and $D$ terms are numbers, only those features of the $A$ and $B$ terms that are associated with numbers allow analogous transformations from the $A$ to the $B$ term.

\section{Six Paradigms for Study}

On the basis of the foregoing analysis, let us define the notions of common feature change and specific feature change as follows. A feature change is said to be in common with the A-B and C-D pairs of terms if the feature under consideration that is changed from $A$ to $B$ can also be found to change from $C$ to $D$. A feature change is specific to $A$ and $B$ if such feature change can be found only from A to B. Similarly, a feature change is specific to $C$ and $D$ if such feature change can be found only from $C$ to $D$.

With verbal analogies, the numbers of common and specific feature changes are very difficult to specify precisely. For this reason, pictorial analogies were used in the present study. For the sake of exposition, let A, B, C, and D stand for the four terms of a pictorialcup analogy, and $P, Q, R$, and $S$ for the four terms of a pictorial-person analogy. It is assumed that there are three feature changes for pictorial cups that are in common with three feature changes for pictorial persons. Furthermore, pictorial cups are assumed to possess two specific feature changes and pictorial persons to have two specific feature changes of their own. With these assumptions, it is possible to construct six paradigms of mixed and unmixed analogies, as follows:

$$
\begin{aligned}
& A: B:: C: D_{1}, D_{2} \\
& P: Q:: R: S_{1}, S_{2} \\
& A: B:: R: S_{1}, S_{2} \\
& P: Q:: C: D_{1}, D_{2} \\
& A: Q:: C: S_{1}, S_{2} \\
& P: B:: R: D_{1}, D_{2} .
\end{aligned}
$$

Paradigms 1 and 2 are unmixed analogies of pictorial cups and pictorial persons, respectively. Paradigms 3 and 4 are mixed analogies in their mapping. Paradigms 5 and 6 are mixed analogies in their inference. The terms mapping and inference have been used to denote the relationships between the first and third terms and between the first and second terms of an analogy, respectively (see Sternberg, 1977).

\section{Predictions from Current Theories}

With these six paradigms, there are two cases to consider. In the first case, there is no specific feature change for any two terms of importance in solving analogies. Such two terms are the first and second terms, the first and third terms, the third and fourth (correct D or S) terms, or the second and fourth terms. In other words, in this case, all pictorial cups of an analogy have identical specific features and all pictorial persons of an analogy have identical specific features also. In the second case, there is at least one specific feature change for two terms of an analogy. In both cases, however, it is assumed that there is one common feature change from the first to second term and one common feature change from the first to third term for each paradigm. 
Let us consider each case separately. For the first case of no specific feature change, some predictions can be made from the existing theories as to the relative magnitudes of solution times for the six paradigms of pictorial analogies. If Paradigms 1 and 2 are combined (called Paradigm 1-2), Paradigms 3 and 4 are combined (called Paradigm 34), and Paradigms 5 and 6 are combined (called Paradigm 5-6), then Paradigms 1-2, 3-4, and 5-6 are equated for the information structure of all concerned individual terms. Differences in the solution times for these three types of paradigms must be attributed to relative difficulties of extracting the feature changes or/and comparing the feature changes. With respect to Paradigm 1-2, comparison of the feature changes, as well as extraction of the feature changes, is made for pictures of the same category. As for Paradigm 3-4, extraction of the feature changes is made for pictures of the same categories, but comparison of the feature changes is made for pictures of different categories. As for Paradigm 5-6, extraction of the feature changes is made for pictures of different categories, and comparison of the feature changes is therefore also made for pictures across different categories. If it is assumed that it is more difficult to compare the feature changes of pictures of one category with those of pictures of another category and that it takes a longer time to extract as well as to compare the feature changes across pictures of different categories than across pictures of the same categories, then the relative magnitudes of solution times for Paradigms 1-2, 3-4, and 5-6 may be characterized by the following inequalities:

$$
\text { Paradigm 1-2 }<\text { Paradigm 3-4 }<\text { Paradigm 5-6. }
$$

As Grudin (1980) observed, if the subject switches to extract the feature changes from the first to the third term and from the second to the fourth term after finding it difficult to extract the feature changes from the first to the second term and from the third to the fourth term in Paradigm 5-6, the subject would be in the same situation as in Paradigm 34. In this case, however, the subject still has spent extra time in attempting to solve analogies of Paradigm 5-6 before the switch. Therefore, solution times for Paradigm 5-6 are still longer than for Paradigm 3-4 and Inequality 7 is unaffected.

A second prediction from the existing theories of analogical reasoning in the case of no specific feature change is symmetries of Paradigms 1 and 2, Paradigms 3 and 4, and Paradigms 5 and 6. Let us consider a symmetry of Paradigms 1 and 2 first. Since there is no specific feature change, all terms in Paradigms 1 and 2 are fixed at some specific features. In order to be comparable, the common feature changes in Paradigm 1 should be the same common feature changes in Paradigm 2. If the extent of each common feature change can approximately be equated for Paradigms 1 and 2, their solution times should not differ substantially unless the solution time for each paradigm depends also on the specific features. With respect to a symmetry of Paradigms 3 and 4 , a common feature change from $A$ to $B$ in Paradigm 3 is equated with a common feature change from $C$ to $D$ (correct fourth term) in Paradigm 4, and a common feature change from $R$ to $S$ (correct fourth term) in Paradigm 3 is equated with a common feature change from $P$ to $Q$ in Paradigm 4. Moreover, the subject compares one common feature change for pictorial cups and one analogous common feature change for pictorial persons in Paradigm 4, as in Paradigm 3. If the solution times depend mainly on extraction and comparison of the feature changes, as asserted by the existing theories of analogical reasoning, then a symmetry of Paradigms 3 and 4 is established. It is to be noted that a symmetry of Paradigms 3 and 4 holds irrespective of a symmetry of Paradigms 1 and 2. By relying on the same reasoning, it can also be shown that Paradigms 5 and 6 are symmetrical so far as the relevant solution processes are concerned.

\section{Predictions from a Theory of Metaphoricity}

A recent theory of metaphoricity, or nonliteral similarity (e.g., Ortony, 1979) has a different view of the symmetry problem. This view takes its departure from Tversky's (1977) theory. The latter is designed to account for the degree of judged similarity between two objects represented by, say, the terms $X$ and $Y$. The theory postulates that the perceived similarity, $S(X, Y)$, is a weighted function of the intersection of features of $X$ and $Y$ (denoted by $F_{X} \cap F_{Y}$ ) less the sum of a weighted function of the features specific to one and a weighted function of the features specific to the other, giving

\section{$S(X, Y)$}

$$
=\theta h\left(F_{X} \cap F_{Y}\right)-\alpha h\left(F_{X}-F_{Y}\right)-\beta h\left(F_{Y}-F_{X}\right) .
$$

In this equation, the function $h$ is a measure of the salience of features and $\theta, \alpha$, and $\beta$ are parameters that reflect the importance of the common and specific features. Ortony modified this equation as follows:

\section{$S(X, Y)$}

$$
=\theta h \mathrm{Y}\left(\mathrm{F}_{\mathrm{X}} \cap \mathrm{F}_{\mathrm{Y}}\right)-\alpha \mathrm{h}^{\mathrm{X}}\left(\mathrm{F}_{\mathrm{X}}-\mathrm{F}_{\mathrm{Y}}\right)-\beta \mathrm{h} \mathrm{Y}\left(\mathrm{F}_{\mathrm{Y}}-\mathrm{F}_{\mathrm{X}}\right),
$$

where $\mathrm{hX}$ and $\mathrm{h}^{\mathrm{Y}}$ represent measures of salience based on the values in $F_{X}$ and $F_{Y}$, respectively.

In translating the language of similarity judgment into the language of analogical reasoning, it is necessary only to replace features by feature changes. It will be clear subsequently that the investigation of cognitive processes in terms of feature changes rather than features alone has an advantage because the contrasting 
features arising from the description of feature changes automatically provide contexts. In dealing with features alone, for example, it is necessary to invoke the concept of diagnosticity (see Tversky, 1977). For the present, since the case of no specific feature change is our main concern, by interpreting a feature as standing for a feature change (denoted by $\mathrm{F}_{\mathrm{X}: \mathrm{Y}}$, etc.), Equation 9 is simplified as follows:

$$
\mathrm{S}(\mathrm{X}: \mathrm{Y}, \mathrm{V}: \mathrm{W})=\theta \mathrm{hV}: \mathrm{W}\left(\mathrm{F}_{\mathrm{X}: \mathrm{Y}} \cap \mathrm{F}_{\mathrm{V}: \mathrm{W}}\right),
$$

where some notational differences are self-explanatory.

In order to see how Equation 10 applies to the symmetry problem under consideration, Paradigm 1 to Paradigm 6 may be rewritten to take the following verbal forms:

A cup is to B cup as C cup is to D cup.

$\mathrm{P}$ person is to $\mathrm{Q}$ person as $\mathrm{R}$ person is to $\mathrm{S}$ person.

$A$ cup is to $B$ cup as $R$ person is to $S$ person.

$P$ person is to $Q$ person as $C$ cup is to $D$ cup.

A cup is to $Q$ person as $C$ cup is to $S$ person.

$P$ person is to $B$ cup as $R$ person is to $D$ cup.

With respect to Forms 1' and 2' (or Paradigms 1 and 2), the feature changes $\left(F_{A: B}, F_{C: D}\right.$ or $\left.F_{P: Q}, F_{R: S}\right)$ to be compared are from the same domain. There is no problem of symmetry because strictly the same feature changes are to be compared in solving analogies of Forms $1^{\prime}$ and $2^{\prime}$. An analogy of this type may be referred to as a literal analogy or literal paradigm. In Forms $3^{\prime}$ and $4^{\prime}$, the feature changes to be compared are from different domains. An analogy of this type may be called a nonliteral analogy or nonliteral paradigm. According to Equation $10, \mathrm{~S}(\mathrm{X}: \mathrm{Y}, \mathrm{V}: \mathrm{W})$ is generally not equal to $S(V: W, X: Y)$, because the salience values of the common feature changes in $\mathrm{V}: \mathrm{W}$ are generally different in $X: Y$ for nonliteral analogies. Therefore, an asymmetry of Forms $3^{\prime}$ and $4^{\prime}$ should be predicted. As for Forms $5^{\prime}$ and $6^{\prime}$, if feature changes can be detected from a cup to a person (Form $5^{\prime}$ ) as easily as from a person to a cup (Form $6^{\prime}$ ), the subject is essentially comparing the same feature changes in determining the existence of analogous relationships. Consequently, a symmetry of Forms $5^{\prime}$ and $6^{\prime}$ should be obtained.

As regards the second case with specific feature changes, let us first consider Paradigms 1 and 2. Since all feature changes take place in the same domain of cups or persons in these paradigms, when a specific feature change is installed from $A$ to $B$ (or $P$ to $Q$ ), the same specific feature change is required from $C$ to $\mathrm{D}$ (or R to S) in order to guarantee the solvability of analogies. Similarly, when a specific feature change is installed from $A$ to $C$, the same specific feature change is also required from B to D. In other words, a specific feature change in the inference or mapping component is not different from a common feature change in the inference or mapping component. A prediction is that addition of a specific feature change in Paradigm 1 or 2 lengthens the solution time, as does addition of a common feature change, as Sternberg (1977) has obtained. However, if the subject attempts to solve analogies of Paradigm 1 or 2 as well as analogies of other paradigms, this lengthening effect of solution time may diminish because he or she may learn to disregard the specific feature changes in solving analogies of other paradigms.

Next, let us consider the effect of a specific feature change in Paradigms 3 and 4. A specific feature change can be placed from $A$ to $B$ without affecting the legitimacy of this analogy, because a feature change that is specific to cups cannot be used to compare with any feature change between persons. Similarly, a feature change that is specific to persons can be installed between $P$ and $Q$ alone in Paradigm 4. A specific feature change cannot be placed between the first and third terms in Paradigms 3 and 4, because they belong to different domains. A second possibility of placing a specific feature change in these paradigms is between the third and fourth terms. In this case, again, the obtained, analogies are legitimate. A prediction of the effect of a specific feature change in Paradigms 3 and 4 can be obtained from extensions of Equation 9, as in the following two equations:

$\mathrm{S}(\mathrm{X}: \mathrm{Y}, \mathrm{V}: \mathrm{W})=$

$\theta \mathrm{hV}: \mathrm{W}\left(\mathrm{F}_{\mathrm{X}: \mathrm{Y}} \cap \mathrm{F}_{\mathrm{V}: \mathrm{W}}\right)-\alpha \mathrm{h} \mathrm{X}: \mathrm{Y}\left(\mathrm{F}_{\mathrm{X}: \mathrm{Y}}-\mathrm{F}_{\mathrm{V}: \mathrm{W}}\right) ;(11)$

$\mathrm{S}(\mathrm{X}: \mathrm{Y}, \mathrm{V}: \mathrm{W})=$

$\theta \mathrm{hV}: \mathrm{W}\left(\mathrm{F}_{\mathrm{X}: \mathrm{Y}} \cap \mathrm{F}_{\mathrm{V}: \mathrm{W}}\right)-\beta \mathrm{hV}: \mathrm{W}\left(\mathrm{F}_{\mathrm{V}: \mathrm{W}}-\mathrm{F}_{\mathrm{X}: \mathrm{Y}}\right)$.

Equation 11 computes magnitude of the analogous relationship when a specific feature change is imposed on the first and second terms, and Equation $12 \mathrm{com}$ putes magnitude of the analogous relationship when a specific feature change is imposed on the third and fourth terms. Since magnitude of the analogous relation. ship is assumed to be inversely related with solution time, an increase in the solution time for Paradigms 3 and 4 may be predicted to depend on the importance of the introduced specific feature change.

Finally, let us consider the effect of introducing a specific feature change in Paradigms 5 and 6. In these paradigms, a specific feature change can be imposed only from the first to the third term or from the second to the fourth term. There are two situations. In the first situation, the subject may attempt to extract feature changes from the first to the second term and from the third to the fourth term for comparison. Then a specific feature change will not be involved at all in the feature changes the subject is attempting to extract. If the 
solution time is affected, it is due to a change in the information structures of individual terms, the effect of which may be considered negligibly small. Therefore, the solution times obtained for Paradigms 5 and 6 should not differ from each other. In the second situation, suppose that the subject proceeds to extract feature changes from the first to the third term and from the second to the fourth term for comparison. Then, this situation is similar to the case of Paradigms 3 and 4.

\section{METHOD}

\section{Subjects}

The subjects were 23 sophomores majoring in psychology. They participated in the experiment to fulfill a laboratory requirement.

\section{Materials and Design}

There were two types of stimuli. Stimuli of the first type were schematic drawings of cups varying on five binary features: size (large/small), direction (upright/upside down), stripe density (densely striped/thinly striped), ear (presence/absence of one ear), and contour (round/cornered). The first three were to be used as common features and the last two as specific features. Stimuli of the second type were schematic drawings of persons varying on five binary features: size (large/small), direction (upright/upside down), stripe density (densely striped/thinly striped), sex (male/female), and socks (wearing/not wearing socks). Again, the first three were common features, the last two, specific features. The pictures were drawn with black ink, xeroxed, and then pasted on tachistoscope cards.

Analogies were forced choice in format. Six paradigms of analogies were constructed. Sample analogies of various paradigms are shown in Figures 1 and 2. For Paradigm 1, all analogy terms were pictorial cups. For Paradigm 2, all analogy terms

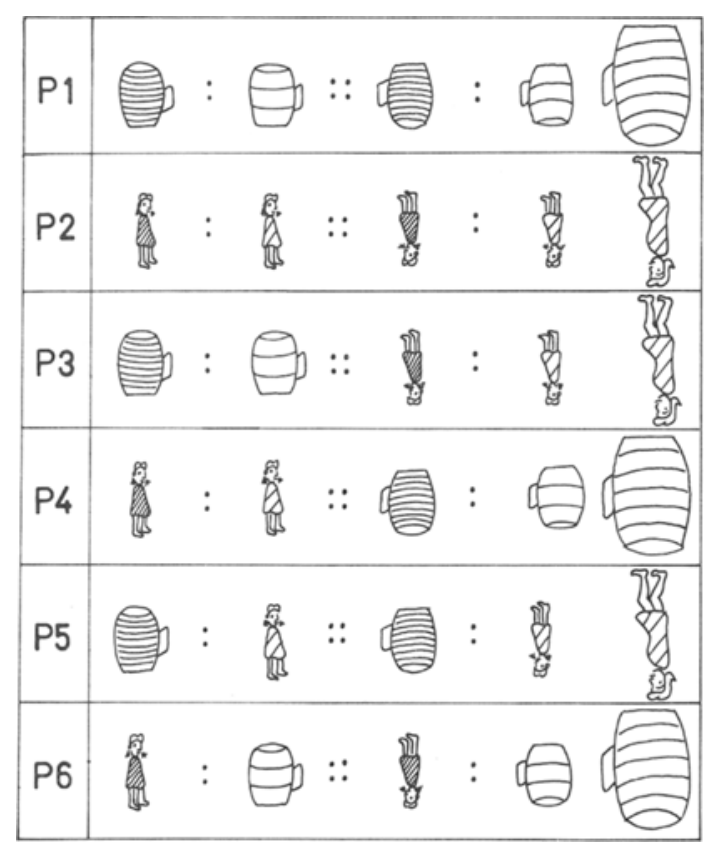

Figure 1. A sample analogy for each paradigm (P) in the case of no specific feature change.

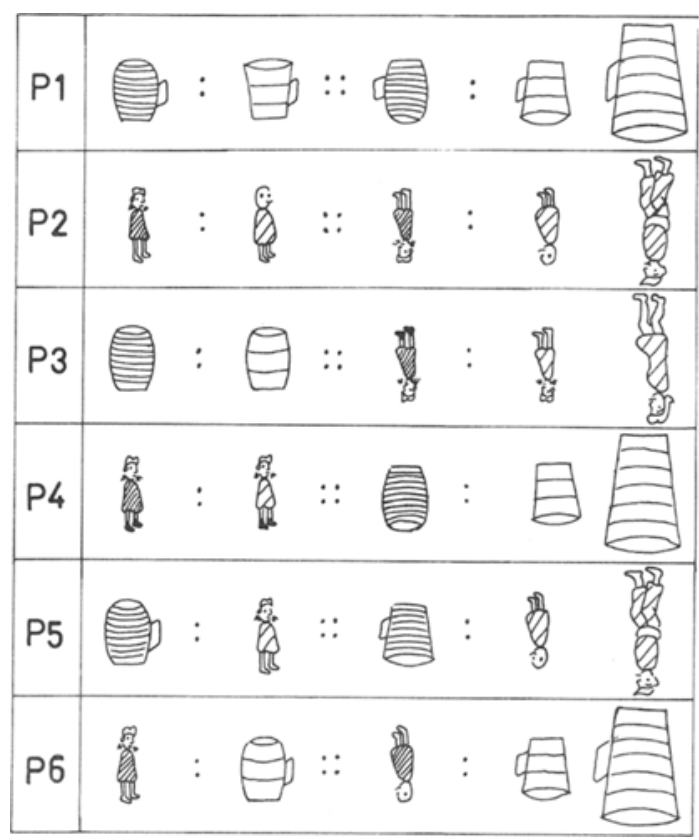

Figure 2. A sample analogy for each paradigm (P) in the cases of imposing a specific feature change in the first to the second term and in the third to alternative terms (P1 and P2) or in the third to alternative terms alone (P3 and P4), or imposing a specific feature change in the first to the third term and another specific feature change in the second to alternative terms (P5 and P6).

were pictorial persons. Paradigm 3 had pictorial cups for the first and second terms and pictorial persons for the remaining terms. Paradigm 4 had pictorial persons for the first and second terms and pictorial cups for the remaining terms. As for Para$\operatorname{digm} 5$, the first and third terms were pictorial cups and the remaining terms, pictorial persons. In the last paradigm, Paradigm 6 , the first and third terms were pictorial persons and the remaining terms, pictorial cups.

With respect to the common features, for each analogy, there was one common feature change from the first to the second term and one common feature change from the first to the third term. This applied to all analogies of all paradigms. A specific feature change could be introduced in two locations for each paradigm. This gave rise to a 2 (locations) by 2 (presence or absence) factorial for each paradigm. The locations of introducing a specific feature change, however, differed from paradigm to paradigm. For Paradigms 1 and 2, a specific feature change might be introduced from the first to the second term or from the first to the third term. For Paradigms 3 and 4 , a specific feature change might be introduced from the first to the second or from the third to the fourth (correct) term. For Paradigms 5 and 6 , a specific feature change might be introduced from the first to the third or from the second to the fourth (correct) term. Figure 1 shows an analogy without any specific feature change for each paradigm. Figure 2 shows an analogy with a specific feature change in the first or/and second locations for each paradigm.

Two alternatives $\left(D_{1}, D_{2}\right.$ or $\left.S_{1}, S_{2}\right)$ were always identical in all specific features but differed in one common feature. For each condition combination of two locations of a specific feature change and two conditions of presence or absence of a specific feature change, four analogies were constructed for each paradigm. Half of these analogies had correct alternatives on the 
right side and the other half on the left side. Altogether, there were 96 analogies.

\section{Procedure}

All 64 individual pictures of cups and persons were drawn systematically on two large sheets of paper. The subject was familiarized with all features involved in these pictures. However, no mention of the common or specific features was made. Twelve practice trials preceded the 96 experimental trials. On each trial, after the experimenter's ready signal, a full analogy was presented on a tachistoscope. The subject pressed the right or left key to indicate his or her choice of the correct alternative. The subject was instructed to respond as fast as possible without making an error. The subject received feedback on each of the 12 practice trials. No feedback was given on the 96 experimental trials.

\section{RESULTS AND DISCUSSION}

The mean solution time obtained under each condition of specific feature change for each paradigm is presented in Table 1 . The percentage of errors for each condition is also listed. There was a tendency for more errors to be associated with longer solution times. In the following, statistical tests of the obtained mean solution times are presented in the order of the problems raised. Therefore, the mean solution times obtained under the condition of no specific feature change is dealt with first. All statistical tests are based on a .05 significance level.

\section{No Specific Feature Change}

For the condition in which no specific feature change was imposed (see sample analogies of Figure 1), the mean solution times of Paradigms 1 and 2 were averaged for each subject. Similarly, such averages were computed for Paradigms 3 and 4 and for Paradigms 5 and 6. The means of these averages for Paradigms 1-2, 3-4, and 5-6 were $2.58,2.64$, and $3.10 \mathrm{sec}$, respectively. The effect of information structure of individual terms was equated in these means. An analysis of variance showed that there was an overall difference among the means $[F(2,44)=$ 13.13, MSe $=.14]$. According to Newman-Keuls tests, the mean solution time was significantly slower in Paradigm 5.6 than in Paradigm 1.2 or $3-4$, but the

Table 1

Mean Solution Times (T) in Seconds and Percentage of Errors (E)

\begin{tabular}{|c|c|c|c|c|c|c|c|c|}
\hline \multirow[b]{3}{*}{$\mathbf{P}$} & \multicolumn{8}{|c|}{ Location of Specific Feature Change } \\
\hline & \multicolumn{2}{|c|}{ None } & \multicolumn{2}{|c|}{ First } & \multicolumn{2}{|c|}{ Second } & \multicolumn{2}{|c|}{$\begin{array}{l}\text { First and } \\
\text { Second }\end{array}$} \\
\hline & $\mathrm{T}$ & $\mathrm{E}$ & $\mathrm{T}$ & $\mathbf{E}$ & $\mathrm{T}$ & $\mathrm{E}$ & $\mathrm{T}$ & $\mathrm{E}$ \\
\hline $\begin{array}{l}1 \\
2 \\
3\end{array}$ & $\begin{array}{l}2.50 \\
2.65 \\
2.40 \\
2.87 \\
2.97 \\
3.22\end{array}$ & $\begin{array}{r}5.4 \\
3.3 \\
10.9 \\
13.0 \\
5.4 \\
7.6\end{array}$ & $\begin{array}{l}2.48 \\
2.57 \\
3.22 \\
2.77 \\
3.14 \\
3.37\end{array}$ & $\begin{array}{r}8.7 \\
5.4 \\
15.2 \\
8.7 \\
3.3 \\
12.0\end{array}$ & $\begin{array}{l}2.54 \\
2.63 \\
3.01 \\
3.06 \\
3.19 \\
3.24\end{array}$ & $\begin{array}{r}18.5 \\
6.5 \\
8.7 \\
6.5 \\
25.0 \\
15.2\end{array}$ & $\begin{array}{l}2.76 \\
2.84 \\
2.97 \\
3.11 \\
3.48 \\
3.19\end{array}$ & $\begin{array}{r}10.9 \\
10.9 \\
10.9 \\
5.4 \\
18.5 \\
20.7\end{array}$ \\
\hline
\end{tabular}

Note $-P=$ paradigm . latter two paradigms did not differ from each other. While this result is in general agreement with Inequality 7 , with the present materials it can be said that comparison of the feature changes for pictures across different categories (cup-cup compared with person-person or person-person compared with cup-cup) was made as effectively as for pictures of "same" categories.

As for the symmetry problem, a question was whether the order of comparing common feature changes has an effect on the solution time. Pairs of mean solution times to be compared are listed in the first column of Table 1 . With respect to Paradigms 1 and 2, the mean solution time of Paradigm 1 was not significantly different from that of Paradigm 2 [t $(22)=1.28]$. However, the mean solution time was slower in Paradigm 4 than in para$\operatorname{digm} 3[t(22)=4.07]$. The mean solution time of Paradigm 5 was found not to differ significantly from that of Paradigm $6[t(22)<1]$.

The obtained asymmetry of Paradigms 3 and 4 is inconsistent with the prediction from the current theories of analogical reasoning, but it is consistent with the prediction from the theory of metaphoricity. Since the mean solution time was faster for Paradigm 3 than for Paradigm 4 in this case, it may be reasoned that common feature changes were more salient in person: person than in cup:cup. This is because the analogous relations obtained for Paradigms 3 and 4 in this case are characterized by the following inequality:

$$
\begin{aligned}
& \text { S(cup:cup,person:person)> } \\
& \text { S(person:person,cup:cup), }
\end{aligned}
$$

which implies a shorter mean solution time for Paradigm 3 than for Paradigm 4.

Before proceeding to the treatment of the data of specific features, it is necessary to make a distinction between two types of features because Sternberg and Rifken (1979) found that which was used made a difference in solution processes. As originally conceived, of the two specific features selected for cups or persons, one was a separable or dissociable feature and the other was an integral or nondissociable feature. A dissociable feature is an attribute of a stimulus that either exists or does not exist, but if it exists, it has only a single level (see Garner, 1974). It is called a dissociable feature because that feature may be taken away from the stimulus without otherwise affecting the rest of the stimulus. A nondissociable feature is an attribute of a stimulus such that if the feature exists for the stimulus. it exists at some positive level and these alternative possible levels are mutually exclusive. In this sense, the ear of cups and the wearing of socks are dissociable features, but the contour of cups and the sex of persons are nondissociable features. However, when a person wearing a pair of socks was drawn in a picture, the result was only a change in color. Therefore, the feature of wearing socks may not be strictly dissociable.

In the condition of no specific feature change, all 
five terms (including a wrong alternative) of an analogy in Paradigms 1 and 2 still stood on the same specific features. Notice that there was only no specific feature change involved. It is then possible to find the effect of type of specific feature on solution time. For Paradigm 1, all analogies may be classified into two categories. In one category, all analogy terms had ears, and in another, all cups were without ears. The mean solution times of these two categories of analogies were 2.78 and $2.21 \mathrm{sec}$, respectively. This difference was significant $[\mathrm{t}(22)=$ 3.49]. Similarly, all analogies in Paradigm 1 may be classified into those of round cups and those of cornered cups. The mean solution times for these two categories were nearly identical, 2.48 and $2.47 \mathrm{sec}$, respectively. With respect to Paradigm 2, in the same way, those analogies with socks took a mean solution time $(2.86 \mathrm{sec})$ longer than those analogies without socks $(2.51 \mathrm{sec})$, although the difference was not significant $[\mathrm{t}(22)=$ 1.49]. When the information structures of individual terms were nearly identical, as in the case of the contour feature in Paradigm 1, those analogies with males as their terms took a mean solution time $(2.62 \mathrm{sec})$ nearly as long as those analogies with females as their terms $(2.67 \mathrm{sec})$. To summarize, the mean solution time obtained for analogies with all terms fixed at one level of a nondissociable feature was nearly identical to that obtained for analogies with all terms fixed at another level, whereas the mean solution time was longer for analogies with all terms possessing a dissociable feature than for analogies with all terms not possessing such a feature. This result apparently reflects the effect of increased complexity in the information structure of individual terms, supporting the finding of Mulholland et al. (1980).

\section{Specific Feature Change}

Since a specific feature change was introduced at different locations for different paradigms, analyses of variance were separately performed on the data obtained with different paradigms.

Paradigm 1-2. According to the first two rows of Table 1, introduction of a single specific feature change seemed to produce no effect on the solution times, although simultaneous application of two specific feature changes tended to increase the solution times in both Paradigms 1 and 2. An analysis of variance performed on the data as represented in the first two rows of Table 1 showed that the difference between the two paradigms was not significant $[F(1,22)=2.35$, $\mathrm{MSe}=.20]$, although Paradigm 2 tended to produce longer solution times under various conditions of introducing specific feature changes. The effects of introducing a specific feature change in the first and second locations were not significant sources of variance $[\mathrm{F}(1,22)<1$ and $\mathrm{F}(1,22)=3.71, \mathrm{MSe}=.25$, respectively].

A question of interest was: What are the effects of dissociable and nondissociable feature changes when they were imposed from the first to the second term or from the first to the third term? As was mentioned previously, since the feature of wearing socks may not be strictly dissociable, a statistical analysis was performed only on the data of Paradigm 1 . The relevant data are in the second and third entries of the first row in Table 1. However, these data represent the combined effects of both dissociable and nondissociable feature changes. The data of the combined effects were reanalyzed, and the mean solution times of interest are presented in the first row of Table 2 . It is to be noted that the number of observations in each column was not necessarily the same because of the randomization procedure. An analysis of variance showed that neither location of specific feature change nor type of specific feature change was a significant source of variance (both with $F<1$ ). However, their interaction was significant $[\mathrm{F}(1,22)=4.71, \mathrm{MSe}=.63]$. The fast mean solution time of $2.28 \mathrm{sec}$ was obtained when a dissociable specific feature change was placed from the first to the third term. In this case, mapping was apparently not used, supporting the finding of Sternberg and Rifkin (1979).

Paradigm 3-4. The relevant data obtained for this paradigm are presented in the third and fourth rows of Table 1. It is clear that no matter where a specific feature change was introduced, the mean solution time was increased considerably in Paradigm 3. As for Para$\operatorname{digm} 4$, the mean solution time was increased only when a specific feature change was introduced from the third to the fourth term. An analysis of variance showed that introduction of a specific feature change from the third to the fourth term increased the mean solution time $[\mathrm{F}(1,22)=7.44, \mathrm{MSe}=.31]$. An increase in the mean solution time was obtained by placing a specific feature change from the first to the second term only in Para$\operatorname{digm} 3$, as reflected in the significant interaction between paradigm and first location of specific feature change $[F(1,22)=6.96, \mathrm{MSe}=.29]$. The three-way interaction was also significant $[\mathrm{F}(1,22)=4.51, \mathrm{MSe}=$ .63]. All other effects were nonsignificant.

An extension of the theory of metaphoricity encounters a difficulty when the above findings obtained

Table 2

Mean Solution Times (T) in Seconds and Percentage Errors (E) Under the Conditions of Dissociable and Nondissociable Specific Feature Changes in Paradigms 1 and 4

\begin{tabular}{|c|c|c|c|c|c|c|c|c|}
\hline \multirow[b]{4}{*}{$\mathbf{P}$} & \multicolumn{8}{|c|}{ Location of Specific Feature Change } \\
\hline & \multicolumn{4}{|c|}{ First } & \multicolumn{4}{|c|}{ Second } \\
\hline & \multicolumn{2}{|c|}{$\begin{array}{c}\text { Dissoci- } \\
\text { able }\end{array}$} & \multicolumn{2}{|c|}{$\begin{array}{l}\text { Nondissoci- } \\
\text { able }\end{array}$} & \multicolumn{2}{|c|}{$\begin{array}{c}\text { Dissoci- } \\
\text { able }\end{array}$} & \multicolumn{2}{|c|}{$\begin{array}{c}\text { Nondissoci- } \\
\text { able }\end{array}$} \\
\hline & $\mathrm{T}$ & $\mathrm{E}$ & $\mathbf{T}$ & $\mathrm{E}$ & $\mathrm{T}$ & $\mathrm{E}$ & $\mathrm{T}$ & $\mathrm{E}$ \\
\hline 1 & 2.73 & 13.4 & 2.29 & 2.1 & 2.28 & 4.3 & 2.60 & 15.6 \\
\hline 4 & 2.50 & 4.3 & 3.01 & 10.9 & 2.82 & 4.3 & 3.22 & 8.7 \\
\hline
\end{tabular}

Note $-P=$ paradigm. 
from introducing specific feature changes are considered. According to Equations 11 and 12 , the effects of introducing specific feature changes in the first to the second term and in the third to the fourth term are uniformly and additively to decrease magnitudes of the analogous relations, $S(A: B, R: S)$ and $S(P: Q, C: D)$ and, in turn, to increase the solution times. Such uniform and additive effects were not obtained. In both Paradigms 3 and 4 , the effect of introducing a specific feature change in the third to alternative terms was unequivocally to increase the mean solution times. The similar effect of introducing a specific feature change in the first to the second term was obtained only for Paradigm 3 . In view of the results obtained for Paradigm 3, $\alpha \mathrm{hX}: \mathrm{Y}\left(\mathrm{F}_{\mathrm{X}: \mathrm{Y}}-\mathrm{F}_{\mathrm{V}: \mathrm{W}}\right)$ of Equation 11 should be about equal to $\beta \mathrm{hV}: \mathrm{W}\left(\mathrm{F}_{\mathrm{V}}: \mathrm{W}-\right.$ $\mathrm{F}_{\mathrm{X}: \mathrm{Y}}$ ) of Equation 12.

It may be interesting to ask which type of specific feature change, dissociable or nondissociable, was responsible for an increase in the mean solution time. In Paradigm 3, as Table 1 shows, the subject made many errors when a specific feature change was introduced from the first to the second term. As a consequence, there were many missing data when the solution times were classified according to different types of specific feature changes for each condition. Hence, an analysis of variance was performed only on the data of Paradigm 4. These data are presented in the second row of Table 2. In Paradigm 3-4, the specific feature changes of cups as well as those of persons were involved. The specific feature change of wearing or not wearing socks is not strictly dissociable. Therefore, the data of the second row in Table 2 are only suggestive. An analysis of variance showed that introduction of a nondissociable specific feature change increased the mean solution time $[F(1,22)=5.66, \quad M S e=.85]$, whereas location of specific feature change failed to reach significance $[F(1,22)=3.57, \mathrm{MSe}=.44]$. Apparently, dissociable feature changes were processed more efficiently.

Paradigm 5-6. The mean solution time obtained under each condition of introducing a specific feature change is listed in the last two rows of Table 1. An analysis of variance showed that the difference between Paradigms 5 and 6 was not significant $(F<1)$. Although introducing a specific feature change in the second location tended to increase the mean solution time only in Paradigm 5, this tendency of interaction was not significant $[F(1,22)=3.72, \quad M S e=.40]$. All other effects were nonsignificant.

\section{A TENTATIVE MODEL}

A model capable of accounting for the obtained results is proposed as follows. This model assumes that the subject solves a nonliteral analogy of Paradigm 3-4 in two stages. In the first stage, the subject makes an overall analysis of individual terms to determine what features to extract and what feature changes to compare. In the second stage of extraction and comparison, the subject extracts feature changes that run from the first to the second term and from the third to the fourth (or an alternative in the forced-choice format) term for comparison to determine the correctness of the fourth term (or the alternative). As the observations of Whitely and Barnes (1979) suggest, there are marked individual differences. Therefore, these two stages may not be completely separable and overlap or even proceed alternately for many subjects.

In the first stage of overall analysis, on the basis of a rudimental analysis of $\mathrm{V}: W$ (considering $X: Y:: V: W$ ), the subject tends to use this result as a reference to consider what possible feature changes are to be expected for $\mathrm{X}: \mathrm{Y}$. This stage is similar to the initial stage of analysis by synthesis (Neisser, 1967), in which the subject forms expectations about the stimulus as a consequence of the results of preceding analyses. The first stage of overall analysis is not required for a literal analogy of Paradigm 1-2. Therefore, as Table 1 shows, the solution times of Paradigm 1-2 are generally shorter than those of Paradigm 3-4, although the presence of the first stage in solving a nonliteral analogy often leads to more efficient solution.

In the second stage, in the case of the forced-choice format, the subject selects a probable fourth term on the basis of the rudimental analysis and makes a serial extraction of feature changes that go from the third to the fourth term. These feature changes are then used as a set of reference feature changes for extracting and comparing feature changes that exist between the first and second terms. In this process, the subject may stop further extraction and comparison of those irrelevant feature changes that may exist between the first and second terms but are not possible members of the set of reference feature changes. Then the subject uses another alternative as the fourth term. The process is repeated for the new fourth term with a new set of reference feature changes. The subject finally selects an alternative that yields a better match between a set of reference feature changes and the feature changes that exist between the first and second term.

In the second stage of extraction and comparison of feature changes, since $\mathrm{V}: \mathrm{W}$ feature changes are used as a set of reference feature changes, some variables that affect the anchoring of reference feature changes naturally influence the efficiency of solution. One such variable is the salience of feature change. If a V:W reference feature change is salient, then it may be easier for the subject to extract an analogous feature change from $X: Y$ and also easier to compare a salient feature change with an analogous feature change. The salience of a feature change obviously depends on the analogy terms used to represent objects. Thus, the three common feature changes used in the present study are more salient for persons than for cups, perhaps because the former are richer in the meanings of these features. A difference in the size for persons represents a growth change, which is an intrinsic change in persons. A difference in the density of stripes for persons represents a difference in clothes or a difference in personalities, 
which is also an intrinsic change in persons. A difference in the direction is very spectacular for persons. All these differences are less genuine for cups.

The present model then implies that, with no specific feature change, the analogies of Paradigm 3 would be solved faster than those of Paradigm 4 , because the reference feature changes were more salient in the former than in the latter. The present model also implies that, when a specific feature change is introduced in $\mathrm{V}: \mathrm{W}$, the nonliteral analogies of Paradigm 3-4 would be solved slower than when no such feature change is introduced, because a set of reference feature changes is expanded to require more extraction and comparison of feature changes. Furthermore, the present model implies that, when a specific feature change is introduced in $\mathrm{X}: \mathrm{Y}$, the nonliteral analogies of Paradigm 3 should be solved slower but those of Paradigm 4 should be solved as fast as when no such specific feature change is introduced. This implication is based on the following reasoning. When a feature change is introduced in $\mathrm{X}: \mathrm{Y}$ of Paradigm 3, the reference feature changes are salient, whereas the specific feature change pertaining to cups $(\mathrm{X}: \mathrm{Y})$ is less salient. Since the reference feature changes are all salient, in the face of processing a less salient feature change in $\mathrm{X}: \mathrm{Y}$, the subject would tend to search for a possibly less salient feature change in $\mathrm{V}: \mathrm{W}$ that is comparable to the one in $\mathrm{X}: \mathrm{Y}$. On the other hand, when a specific feature change is introduced in $\mathrm{X}: \mathrm{Y}$ of Paradigm 4, the reference feature changes are less salient, and the specific feature change pertaining to persons $(X: Y)$ is salient. Since the reference feature changes are already less salient, in the face of a salient specific feature change in $\mathrm{X}: \mathrm{Y}$, it is as if the subject has exhausted all possible reference feature changes for comparison and, hence, would tend to ignore the salient specific feature change in $\mathrm{X}: \mathrm{Y}$ for further processing. Therefore, the obtained solution time would be as when there is no specific feature change in $\mathrm{X}: \mathrm{Y}$. All these implications are in agreement with the results obtained in the present experiment.

When the reference feature changes of $\mathrm{V}: \mathrm{W}$ are equally as salient as those of $\mathrm{X}: \mathrm{Y}$, we have literal analogies comparable to those of Paradigm 1-2. Without an asymmetry of the salience values for the feature changes of $\mathrm{X}: \mathrm{Y}$ and $\mathrm{V}: \mathrm{W}$, neither the feature changes of $\mathrm{V}: \mathrm{W}$ nor those of $\mathrm{X}: \mathrm{Y}$ may be used as a set of reference feature changes. In this situation, the subject freely compares one feature change of $X: Y$ with an analogous feature change of $\mathrm{V}: \mathrm{W}$ without using either as a reference. This advantage of not relying on a set of feature changes as a reference has a drawback, because the $\mathrm{X}$ and $\mathrm{V}$ terms are generally different. In other words, in the symmetry situation, it is easier to compare the feature changes of $\mathrm{X}: \mathrm{Y}$ with those of $\mathrm{V}: \mathrm{W}$ only when the $V$ term differs from the $X$ term in fewer number of features. Thus, the mapping component becomes important.

It can now clearly be seen that Sternberg's (1977) model applies to a special case of literal analogies.
Mainly based on the results obtained from peoplepiece analogies, Sternberg found that solution time varied with the number of features by which the third term differed from the first term. To account for this effect, he postulated a mapping process from the first to third term, similar to the inference of a rule connecting the first and second terms.

Even for literal analogies, there is a certain condition that favors anchoring the $\mathrm{V}: \mathrm{W}$ feature changes to be used as the reference feature changes. Such a condition is obtained when the $\mathrm{V}$ and $\mathrm{W}$ terms are differentiated on the basis of dissociable features because the $\mathrm{V}$ term is then dissociably distinguishable from the $\mathrm{W}$ term. In other words, the $\mathrm{V}$ term can be distinguished from the $W$ term on the basis of some separable element. In support of this argument, Sternberg and Rifkin (1979) found that mapping is not required for schematicpicture analogies with dissociable features. With respect to the literal analogies of Paradigm 1, when a specific dissociable feature change was imposed from the first to the third term, the subject solved faster than when a specific nondissociable feature change was used. Finally, one implication of the present model for building a theory of metaphoricity as well as a theory of similarity judgment may briefly be noted. Ortony (1979) clearly recognized that it is difficult to build a theory of metaphoricity from below, that is, from a theory of similarity judgment alone. With a theory of analogous relations, a starting reference point is specified in advance and a theory of metaphoricity as well as a theory of similarity judgment may follow naturally.

\section{REFERENCES}

Evans, T. G. A program for the solution of geometric-analogy intelligence test questions. In M. Minsky (Ed.), Semantic information processing. Cambridge, Mass: M.I.T. Press, 1968.

GARNER, W. R. The processing of information and structure. Potomac, Md: Erlbaum, 1974.

Grudin, F. Processes in verbal analogy solution. Journal of Experimental Psychology: Human Perception and Performance, $1980,6,67-74$.

Mulholland, T. M., Pellegrino, J. W., \& Glaser, R. Components of geometric analogy solution. Cognitive Psychology, 1980, 12, 252-284.

NeISSER, U. Cognitive psychology. New York: Appleton-CenturyCrofts, 1967.

Ortony, A. Beyond literal similarity. Psychological Review, 1979, 86, 161-180.

Sternberg, R. J. Component processes in analogical reasoning. Psychological Review, 1977, 84, 353-378.

Sternberg, R. J., \& Rifkin, B. The development of analogical reasoning processes. Journal of Experimental Child Psychology, $1979,27,195-232$.

Tversky, A. Features of similarity. Psychological Review, 1977, 84, 327-352.

Whitely, S. E., \& Barnes, G. M. The implications of processing event sequences for theories of analogical reasoning. Memory \& Cognition, 1979, 7, 323-331.

(Received for publication November 17, 1980; revision accepted April 10, 1981.) 